\title{
MINIMAL ENTROPY FOR ENDOMORPHISMS OF THE CIRCLE
}

\author{
RYUICHI ITO
}

\begin{abstract}
Let $f$ be an endomorphism (continuous map) of the circle which has two periodic points of period $m$ and $n$ respectively such that $m \geq 2, n \geq 2$ and $(m, n)=1$, then topological entropy $h(f) \geq \log \mu_{m, n}$ where $\mu_{m, n}$ is the largest zero of the polynomial $x^{m+n}-x^{m}-x^{n}-1$.
\end{abstract}

Introduction. In [3], L. Block, J. Guckenheimer, M. Misiurewicz and L. S. Young gave, among other things, minimal topological entropy for several types of endomorphisms (continuous maps) of the circle which have a fixed point and another periodic point. Main results in [3] will be shown later as Lemmas 2.1 and 2.4 without proof. L. Block, E. M. Coven and Z. Nitecki [2] have given some improved estimates of minimal entropy after [3]. The aim of this paper is to prove the following.

THEOREM B. Let $f$ be an endomorphism of the circle which has two periodic points of period $m$ and $n$ respectively such that $m \geq 2, n \geq 2$ and $(m, n)=1$. Then topological entropy $h(f) \geq \log \mu_{m, n}$ where $\mu_{m, n}$ is the largest zero of $x^{m+n}-x^{m}-$ $x^{n}-1$.

Notice that $f$ has no condition on degree. We also give an example of endomorphism which attains the smallest possible entropy $\log \mu_{m, n}$.

Let $R$ denote the real numbers, $Z$ the integers, $N$ the positive integers, and $S=$ $R / Z$ the circle. Let $\pi: R \rightarrow S$ denote the canonical projection. For simplicity, we will often write $x$ instead of $\pi(x)$ for $0 \leq x<1$. Let $f: S \rightarrow S$ be an endomorphism of degree $k$. Choose a lifting $F: R \rightarrow R$, that is a map such that $\pi F=f \pi$. Liftings exist and are unique up to the addition of an integer. Each lifting satisfies $F(x+1)=F(x)+k$.

DEFINITION. Let $f$ be an endomorphism of degree 1 and $F$ be a lifting of $f$. We define the rotation number

$$
\rho(F, x)=\limsup _{n \rightarrow \infty} \frac{1}{n}\left(F^{n}(x)-x\right)
$$

and the rotation set

$$
\rho(F)=\{\rho(F, x): x \in R\}=\{\rho(F, x): x \in[0,1)\} .
$$

Notice that if a different lifting is used, then this simply has the effect of translating the rotation number and set by an integer.

It is known (see [4 or 6]) that $\rho(F)$ is a single point or a closed interval, and if $p / m \in \rho(F)$ and $(p, m)=1$ then $f$ has a periodic point $a$ of period $m$ with $\rho(F, a)=$

Received by the editors July 31, 1981 and, in revised form, November 2, 1981.

1980 Mathematics Subject Classification. Primary 58F20.

Key words and phrases. A-graph, loop, periodic point, rotation set, topological entropy. 
$p / m$. Conversely if $f$ has a periodic point of period $m$ then there is $l \in Z$ such that $l / m \in \rho(F)$. Moreover it is easy to see that if $F$ is monotone then $\rho(F)$ is a single point.

We remark here that in [1] C. Bernhardt gave relations among rotation set, topological entropy and, so-called, twist number for the set of endomorphisms of degree 1 having one maximum and one minimum, to which the one attaining the smallest entropy in the example belongs.

1. Let $f$ be an endomorphism of $S$ of degree 1. By an orbit of periodic points of $f$ of period $m$, we mean a set $\left\{a_{0}, \ldots, a_{m-1}\right\}=\left\{f^{i}\left(a_{0}\right): i=0, \ldots, m-1\right\}$ such that $f^{m}\left(a_{0}\right)=a_{0}$ and $0 \leq a_{0}<a_{1}<\cdots<a_{m-1}<1$ in $S$. We define $a_{k}=a_{i}+u$ in $R$ if $a_{i}$ is in the orbit of period $m, k=m u+i, k, u, i \in Z$ and $0 \leq i \leq m-1$, and use this notation throughout this paper.

LEMMA 1.1. Let $f$ have an orbit $\left\{a_{0}, \ldots, a_{m-1}\right\}$ of periodic points of period $m \geq 2$ and have no periodic points of period less than $m$. Then $a_{k}<a_{l}$ implies $F\left(a_{k}\right)<F\left(a_{l}\right)$ where $a_{k}$ and $a_{l}$ are defined as above for $k$ and $l \in Z$.

Proof. By definition $a_{k}<a_{l}$ if $k<l$. Suppose $F\left(a_{s}\right)>F\left(a_{t}\right)$ for $a_{s}<a_{t}$, then there exists $j$ such that $F\left(a_{j-1}\right)<F\left(a_{j}\right)$ and $F\left(a_{j}\right)>F\left(a_{j+1}\right)$. Let $I_{k}=\left[a_{k}, a_{k+1}\right]$, $k \in Z$, be intervals on $R$. As $F\left(a_{j-1}\right) \neq F\left(a_{j+1}\right)$, there exists two adjacent intervals, say $I_{l-1}$ and $I_{l}$, such that $F\left(I_{j-1}\right) \cap F\left(I_{j}\right) \supset I_{l}$ and either $F\left(I_{j-1}\right) \supset I_{l-1}$ or $F\left(I_{j}\right) \supset$ $I_{l-1}$. Since we have only $m$ different intervals $I_{0}, \ldots, I_{m-1}$ on $S$, a sequence $I_{i_{1}} \rightarrow$ $I_{i_{2}} \rightarrow \cdots \rightarrow I_{i_{m-1}}$ of $m-1$ intervals on $S$ such that $f\left(I_{i_{\alpha}}\right) \supset I_{i_{\alpha+1}}$ and $I_{i_{1}}=\pi\left(I_{l}\right)$, either includes one of $\pi\left(I_{j-1}\right)$ and $\pi\left(I_{j}\right)$ or has some interval, say $I_{r}$, twice. The first case gives the sequence $\pi\left(I_{l}\right) \rightarrow \cdots \rightarrow \pi\left(I_{j-1}\right)$ or $\pi\left(I_{j}\right) \rightarrow \pi\left(I_{l}\right)$ so that we have $f^{\sigma}\left(\pi\left(I_{l}\right)\right) \supset \pi\left(I_{l}\right)$ for some $\sigma \leq m-1$. The second case implies $f^{\tau}\left(I_{r}\right) \supset I_{r}$ for some $\tau \leq m-2$. Therefore we have a periodic point of period less than $m$ in each case, contradicting the hypothesis.

Let $A_{m}=\left\{a_{0}, \ldots, a_{m-1}\right\}$ and $B_{n}=\left\{b_{0}, \ldots, b_{n-1}\right\}$ be two orbits of periodic points of $f$ of period $m$ and $n$ respectively. Assume $\rho\left(F, a_{i}\right) \neq \rho\left(F, b_{j}\right)$ for a lifting $F$ of $f$. Let $\hat{F}: R \rightarrow R$ be a mapping such that the graph of $\hat{F}$ is made of line segments connecting $(c, F(c))$ and $(d, F(d))$ where $c$ and $d$ are adjacent points among the set $\left\{a_{k}: k \in Z\right\} \cup\left\{b_{l}: l \in Z\right\}$ in $R$. Let $\hat{f}$ be the endomorphism of $S$, of which $\hat{F}$ is a lifting. In fact $\hat{f}$ is $\pi \hat{F} \mid[0,1]$ and of degree 1 . We call $\hat{F}$ and $\hat{f}\langle m, n\rangle$-skeletons of $F$ and $f$ respectively with respect to $A_{m}$ and $B_{n}$. By the manner of constructing $\hat{f}$ from $f$, we have $h(\hat{f}) \leq h(f)$ (see $[\mathbf{5}]$ ).

DEFINITION. $\langle m, n\rangle$-skeletons $\hat{F}$ and $\hat{f}$ with respect to $A_{m}$ and $B_{n}$ are called simple if $\hat{F}$ keeps the order among $\left\{a_{k}: k \in Z\right\}$, and if

(1) $\rho\left(\hat{F}, a_{i}\right)>\rho\left(\hat{F}, b_{j}\right)$ and $\hat{F}$ does not have a local maximum at any $b_{j}$, or

(2) $\rho\left(\hat{F}, a_{i}\right)<\rho\left(\hat{F}, b_{j}\right)$ and $\hat{F}$ does not have a local minimum at any $b_{j}$.

Hereafter we often assume the following hypothesis for $f$ and say $f$ satisfies $H_{m, n}$

$\left(H_{m, n}\right):$ (I) $f$ is an endomorphism of $S$ of degree 1 .

(II) $f$ has two orbits of periodic points $A_{m}=\left\{a_{0}, \ldots, a_{m-1}\right\}$ and $B_{n}=\left\{b_{0}, \ldots, b_{n-1}\right\}$ such that $\rho\left(F, a_{0}\right) \neq \rho\left(F, b_{0}\right)$ for a lifting $F$ of $f$.

The next lemma will be used only in the proof of Lemma 1.3.

LEMMA 1.2. Let $F$ and $G$ be liftings of endomorphisms $f$ and $g$ of degree 1 respectively. Assume that for any $x \in R$ there exists $y \in R$ such that $y \geq x$ and 
$F(x) \geq G(y)$. Then $\rho^{-}(F) \geq \rho^{-}(G)$ where $\rho^{-}(F)$ denotes the largest lower bound of the rotation set $\rho(F)$.

PROOF. First we prove by induction on $n$ that for any $x \in R$ there exists $y_{n}$ such that $y_{n} \geq x$ and $F^{n}(x) \geq G^{n}\left(y_{n}\right)$. For $n=1$, this is just the assumption of the lemma. Assume that it is true for $n$. Then there exists $u \in R$ such that $u \geq F(x)$ and $F^{n+1}(x)=F^{n}(F(x)) \geq G^{n}(u)$. Since there is $y_{1}$ such that $y_{1} \geq x$ and $F(x) \geq G\left(y_{1}\right)$, and $G(x+p)=G(x)+p$ for $p \in Z$, we have $y_{n+1} \in R$ such that $y_{n+1} \geq y_{1}$ and $G\left(y_{n+1}\right)=u$. This $y_{n+1}$ satisfies $y_{n+1} \geq x$ and $F^{n+1}(x) \geq G^{n}(u)=$ $G^{n}\left(G\left(y_{n+1}\right)\right)=G^{n+1}\left(y_{n+1}\right)$, completing the induction.

To prove $\rho^{-}(F) \geq \rho^{-}(G)$, it suffices to show $\rho^{-}(G) \leq k / n$ for any rational number $k / n \geq \rho^{-}(F)$. Assume $\rho^{-}(F) \leq k / n$. Then we have either $a \in[0,1]$ such that $F^{n}(a)=a+k$ or $F^{n}(x)<x+k$ for any $x \in[0,1]$. In the first case we have $b \in R$ such that $b \geq a$ and $G^{n}(b) \leq F^{n}(a)=a+k \leq b+k$. In the second case we have $c$ such that $c \geq 1$ and $G^{n}(c) \leq F^{n}(1)<1+k \leq c+k$. Since $G^{n}(x+p)=G^{n}(x)+p$ for $p \in Z$, in both cases, we have either $y \in[0,1]$ such that $G^{n}(y)=y+k$ or $G^{n}(x)<x+k$ for any $x$. Therefore we have $\rho^{-}(G) \leq k / n$, completing the proof.

LEMMA 1.3. Let $f$ satisfy $H_{m, n}$ where $1<m \leq n$ and have no periodic points of period less than $m$. Then there exists a piecewise linear endomorphism $g$ of $S$ of degree 1 satisfying the following.

(1) $g$ satisfies $H_{m, n}$, for some $n^{\prime}$ such that $1<n^{\prime} \leq n$,

(2) $g$ is a simple $\left\langle m, n^{\prime}\right\rangle$-skeleton of itself,

(3) $g$ has no fixed point,

(4) $h(g) \leq h(f)$.

ProOF. We may assume $\rho\left(F, a_{0}\right)>\rho\left(F, b_{0}\right)$, because the case $\rho\left(F, a_{0}\right)<\rho\left(F, b_{0}\right)$ goes similarly. If $\hat{f}$, skeleton of $f$, is simple then we have nothing to prove. Let $\hat{f}$ be not simple. Since $\hat{F}$ preserves the order among $\left\{a_{k}: k \in Z\right\}$ by Lemma 1.1, $\hat{F}$ has a local maximum at some $b_{j}$. Take, say $c_{s}$ and $c_{t}$ from the set $\left\{a_{k}: k \in Z\right\} \cup\left\{b_{l}: l \in\right.$ $Z\}$ such that $c_{s}<b_{j}<c_{t}$ and no element of $\left\{a_{k}: k \in Z\right\} \cup\left\{b_{l}: l \in Z\right\}$ is in $\left(c_{s}, c_{t}\right)$ except $b_{j}$. We modify the graph of $\hat{F}$ on $\left[c_{s}, c_{t}\right]$ into the line segment connecting $\left(c_{s}, F\left(c_{s}\right)\right)$ and $\left(c_{t}, F\left(c_{t}\right)\right)$. By doing similar modification on all the intervals of type $\left[c_{s}+u, c_{t}+u\right], u \in Z$, we have a mapping $F_{1}: R \rightarrow R$ whose graph is constructed by these modifications from the graph of $\hat{F}$. If $F_{1}$ still has a local maximum at some $b_{j^{\prime}}$, then we do similar modifications around $b_{j^{\prime}}+u, u \in Z$, as above. Since $\left\{b_{0}, \ldots, b_{n-1}\right\}$ is a finite set, eventually we have a mapping $F^{\prime}$ which does not have a local maximum at any point of $\left\{b_{l}: l \in Z\right\}$. Let $f^{\prime}=\pi F^{\prime} \mid[0,1)$, then $F^{\prime}$ is a lifting of $f^{\prime}$ and $f^{\prime}$ still has the orbit $\left\{a_{0}, \ldots, a_{m-1}\right\}$. Since $F(x) \geq F^{\prime}(x)$, we have $\rho^{-}(F) \geq$ $\rho^{-}\left(F^{\prime}\right)$ by Lemma 1.2. Thus we have $\rho\left(F^{\prime}\right) \supset\left[\rho^{-}(F), \rho\left(F, a_{0}\right)\right] \supset\left[\rho\left(F, b_{0}\right), \rho\left(F, a_{0}\right)\right]$. Therefore we have an orbit of periodic points of $f^{\prime},\left\{b_{0}^{\prime}, \ldots, b_{n^{\prime}}^{\prime}\right\}$ such that $n^{\prime} \leq$ $n$ and $\rho\left(F^{\prime}, b_{0}^{\prime}\right)<\rho\left(F^{\prime}, a_{0}\right)$. Let $\hat{f}^{\prime}$ and $\hat{F}^{\prime}$ be the $\left\langle m, n^{\prime}\right\rangle$-skeletons of $f^{\prime}$ and $F^{\prime}$ respectively with respect to $\left\{a_{0}, \ldots, a_{m-1}\right\}$ and $\left\{b_{0}^{\prime}, \ldots, b_{n^{\prime}-1}^{\prime}\right\}$. By assumption and the manner of constructing $\hat{f}^{\prime}, \hat{f}^{\prime}$ has no fixed point. Any local maximum of $F^{\prime}$ is at some $a_{i}$ and $F^{\prime}$ preserves the order among $\left\{a_{k}: k \in Z\right\}$. Moreover $\rho\left(\hat{F}^{\prime}, b_{0}^{\prime}\right)=$ $\rho\left(F^{\prime}, b_{0}^{\prime}\right)<\rho\left(F^{\prime}, a_{0}\right)=\rho\left(\hat{F}^{\prime}, a_{0}\right)$. Thus $\hat{f}^{\prime}$ satisfies (1), (2) and (3). By the manner of constructing $\hat{f}^{\prime}$ from $f, g=\hat{f}^{\prime}$ also satisfies (4) (see [5]). 
Let $f$ satisfy $H_{m, n}$ with respect to $A_{m}=\left\{a_{0}, \ldots, a_{m-1}\right\}$ and $B_{n}=\left\{b_{0}, \ldots\right.$, $\left.b_{n-1}\right\}$. Let $C=\left\{c_{0}, \ldots, c_{m+n-1}\right\}=A_{m} \cup B_{n}$ and $0 \leq c_{0}<\cdots<c_{m+n-1}$ on $S$. Let $c_{\varsigma}=c_{\eta}+w$ for $\varsigma=w(m+n)+\eta, \zeta, \eta, w \in Z$ and $0 \leq \eta \leq m+n-1$.

Let $I_{\eta}=\left[c_{\eta}, c_{\eta+1}\right], \eta=0, \ldots, m+n-2$, and $I_{m+n-1}=\left[c_{m+n-1}, c_{0}\right]$ be intervals on $S$. We say $I_{\xi} f$-covers $I_{\eta} p$ times if there exist subintervals $K_{1}, \ldots, K_{p}$ of $I_{\xi}$ with pairwise disjoint interiors such that $f\left(K_{i}\right)=I_{\eta}$ for $i=1, \ldots, p$.

DEFINITION. An $A$-graph of $f$ with respect to $A_{m}$ and $B_{n}$ is an oriented graph with vertices $I_{1}, \ldots, I_{m+n-1}$ such that if $I_{\xi} f$-covers $I_{\eta} p$ times but not $p+1$ times then there are $p$ arrows from $I_{\xi}$ to $I_{\eta}$. A sequence $I_{i_{0}} \rightarrow \cdots \rightarrow I_{i_{r}}$ in an $A$-graph of $f$ is called a path of length $r$, and the path is called a loop if $I_{i_{0}}=I_{i_{r}}$. A loop is called simple if $I_{i_{\alpha}} \neq I_{i_{\beta}}$ for $0 \leq \alpha<\beta<r$.

LEMMA 1.4. Let $f$ satisfy $H_{m, n}$ where $1<m$ and $1<n$, have no fixed points and be the simple $\langle m, n\rangle$-skeleton of itself. Then the $A$-graph of $f$ with respect to $A_{m}$ and $B_{n}$ has three different simple loops $L_{1}, L_{2}$, and $L_{3}$ through a vertex, say $J$, of length $l_{1}, l_{2}$ and $l_{3}$ such that $l_{1} \leq m, l_{2} \leq n$ and $l_{3} \leq m+n$, and the last vertices before $J$ of $L_{1}, L_{2}$ and $L_{3}$ are all different.

Proof. We may assume $\rho\left(F, a_{0}\right)>\rho\left(F, b_{0}\right)$ for a lifting $F$ of $f$. Let $F$ have a local maximum at $a_{i}$. Let $b_{j}$ be the adjacent one of $a_{i}$ to the right $\left(a_{i}<b_{j}\right)$ among $\left\{a_{k}: k \in Z\right\} \cup\left\{b_{l}: l \in Z\right\}$. Then $F\left(a_{i}\right)>F\left(b_{j}\right)$ and there exists $a_{s}$ and $b_{t}$ such that $F\left(a_{i}\right) \geq a_{s}>b_{t} \geq F\left(b_{j}\right),\left(b_{t}, F\left(a_{i}\right)\right] \cap\left\{b_{l}: l \in Z\right\}=\varnothing$ and $\left[b_{t}, a_{s}\right) \cap\left\{a_{k}: k \in\right.$ $Z\}=\varnothing$. And we note $a_{i+1}=\inf \left\{a_{k}: a_{k}>b_{j}\right\}$ and $b_{j-1}=\sup \left\{b_{l}: b_{l}<a_{i}\right\}$. Since $\left\{a_{0}, \ldots, a_{m-1}\right\}$ are periodic and $f$ has no fixed point, there exists $\alpha \leq m-1$ such that $F^{\alpha}\left(a_{s}\right)=a_{i}+v$ for some $v \in Z$. Since $f$ is simple and $\rho\left(F, a_{0}\right)>\rho\left(F, b_{0}\right), b_{l}<$ $a_{k}$ implies $F\left(b_{l}\right)<F\left(a_{k}\right)$. Thus we have $F^{\alpha}\left(b_{t}\right)<a_{i}+v$ and so $F^{\alpha}\left(b_{t}\right) \leq b_{j-1}+v$. Let $J=\pi\left(\left[b_{t}, a_{s}\right]\right)$, then $f^{\alpha}(J) \supset \pi\left(\left[b_{j-1}, a_{i}\right]\right)$ and $f\left(\pi\left(\left[b_{j-1}, a_{i}\right]\right)\right) \supset J$. On the other hand, we have $F^{\beta}\left(b_{t}\right)=b_{j}+w$ for some $\beta \leq n-1$ and $w \in Z$. Thus $F^{\beta}\left(a_{s}\right)>b_{j}+w$ and $F^{\beta}\left(a_{s}\right) \geq a_{i+1}+w$. Therefore $f^{\beta}(J) \supset \pi\left(\left[b_{j}, a_{i+1}\right]\right)$ and $f\left(\pi\left(\left[b_{j}, a_{i+1}\right]\right)\right) \supset J$.

Now we have two different intervals say $I_{p} \subset \pi\left(\left[b_{j-1}, a_{i}\right]\right)$ and $I_{q} \subset \pi\left(\left[b_{j}, a_{i+1}\right]\right)$ such that $f^{\alpha}(J) \supset I_{p}, f\left(I_{p}\right) \supset J, f^{\beta}(J) \supset I_{q}$ and $f\left(I_{q}\right) \supset J$ where $0<\alpha \leq m-1$ and $0<\beta \leq n-1$. Furthermore we have another interval $I_{r}=\pi\left(\left[a_{i}, b_{j}\right]\right)$ satisfying $f\left(I_{r}\right) \supset J$. As $\rho\left(F, a_{s}\right) \neq \rho\left(F, b_{t}\right)$, we have $f^{\sigma}(J) \supset I_{r}$ for some $\sigma \in N$ large enough. Since we have only $m+n$ different intervals on $S$, there exists $0<\gamma \leq m+n-1$ such that $f^{\gamma}(J) \supset I_{r}$. Therefore we have three different loops of length $\alpha+1, \beta+1$ and $\gamma+1$ from $J$ to $J$. If any of them is not simple, then it is easy to see we can get an even shorter loop which is simple and has the same last vertex before $J$. Consequently the $A$-graph of $f$ has three simple loops which satisfy the statement of the lemma.

LEMMA 1.5. Let $f$ satisfy $H_{m, n}$ and the A-graph of $f$ with respect to $A_{m}$ and $B_{n}$ have three simple loops $L_{1}, L_{2}$ and $L_{3}$ through a vertex $J$ of length $l_{1}, l_{2}$ and $l_{3}$ respectively where $l_{1} \leq l_{2} \leq l_{3}$. Assume the last vertices $I_{p}, I_{q}$ and $I_{r}$ of $L_{1}, L_{2}$ and $L_{3}$ before $J$ are all different, and that $L_{1}, L_{2}$ and $L_{3}$ are the shortest loops of all the loops through $J$ whose last vertices are $I_{p}, I_{q}$ and $I_{r}$ respectively. Then the A-graph of $f$ has a subgraph which has just three simple loops $L_{1}, L_{2}^{\prime}$ and $L_{3}^{\prime}$ all through $J$ of length $l_{1}, l_{2}$ and $l_{3}$ respectively such that the last vertices of $L_{2}^{\prime}$ and $L_{3}^{\prime}$ before $J$ are $I_{q}$ and $I_{r}$ respectively.

Proof. If $L_{2}$ does not intersect with $L_{1}$ at vertices other than $J$, then let $L_{2}^{\prime}=L_{2}$. If $L_{2}$ intersects with $L_{1}$, let $I_{s} \neq J$ be the last vertex of $L_{2}$ that is also 
on $L_{1}$. Let $L_{2}^{\prime}$ be the loop which is on $L_{1}$ from $J$ to $I_{s}$ and is on $L_{2}$ from $I_{s}$ to $J$. Because of the shortness of $L_{1}$ and $L_{2}$, the length of $L_{2}^{\prime}$ is $l_{2}$. If $L_{3}$ does not intersect with neither $L_{1}$ nor $L_{2}^{\prime}$ outside of $J$, let $L_{3}^{\prime}=L_{3}$. If $L_{3}$ intersects with $L_{1}$ or $L_{2}^{\prime}$, let $I_{t} \neq J$ be the last vertex of $L_{3}$ that is also on $L_{1}$ or $L_{2}^{\prime}$. Let $L_{3}^{\prime}$ be the loop which is on either $L_{1}$ or $L_{2}^{\prime}$ from $J$ to $I_{t}$ depending on whether $I_{t}$ is on $L$ or on $L_{2}^{\prime}$, and is on $L_{3}$ from $I_{t}$ to $J$. Again by the shortness of $L_{1}, L_{2}^{\prime}$ and $L_{3}$, the length of $L_{3}^{\prime}$ is $l_{3}$. Therefore the subgraph consisting of $L_{1}, L_{2}^{\prime}$ and $L_{3}^{\prime}$ satisfies the requirement.

Let $C$ be an $A$-graph of $g$ and $s$ be the number of vertices of $G$. Following [3], we associate to $G$ an $s \times s$ matrix $M=\left(m_{i j}\right)$ such that $m_{i j}=$ (number of arrows from $I_{i}$ to $I_{j}$ ). We call the logarithm of the spectral radius (i.e. of the largest eigenvalue) of $M$ the entropy of $G$ and denote it by $h(G)$.

LEMMA 1.6 [3, LEMMA 1.5]. If $G$ is an A-graph of $g$, then $h(G) \leq h(g)$.

Since $h(G)$ is the limit of $\frac{1}{n} \log$ (sum of entries of $M^{n}$ ) the following is trivial.

LEMMA 1.7. Let $G^{\prime}$ be a subgraph of $G$, then $h\left(G^{\prime}\right) \leq h(G)$.

As a special case of Theorem 1.7 of [3] we have the following.

LEMMA 1.8. Let $G$ be a graph consisting of three different simple loops $L_{1}, L_{2}$ and $L_{3}$ all through a vertex $J$ of length $l_{1}, l_{2}$ and $l_{3}$ respectively. Assume $G$ has no other simple loops. Let $M$ be the matrix associated to $G$. Then the characteristic polynomial of $M$ is $(-1)^{s}\left(x^{s}-x^{s-l_{1}}-x^{s-l_{2}}-x^{s-l_{3}}\right)$, where $s$ is the number of vertices of $G$.

The next lemma is easily proved by direct calculation or by application of Lemma 1.8 of [3].

LEMMA 1.9. Let $m, n, l_{1}, l_{2}$ and $l_{3}$ be positive integers such that $l_{1} \leq l_{2} \leq l_{3}, l_{1} \leq$ $m, l_{2} \leq n$ and $l_{3} \leq m+n$. Let $\mu_{m, n}$ and $\nu$ be the largest zeros of $x^{m+n}-x^{m}-x^{n}-1$ and of $x^{l_{3}}-x^{l_{3}-l_{1}}-x^{l_{3}-l_{2}}-1$ respectively. Then

(1) $\mu_{m, n} \leq \nu$,

(2) if $1 \leq m^{\prime} \leq m$ and $1 \leq n^{\prime} \leq n$, then $\mu_{m, n} \leq \mu_{m^{\prime}, n^{\prime}}$.

THEOREM A. Let $f$ satisfy $H_{m, n}$. Then $h(f) \geq \log \mu_{m, n}$ where $\mu_{m, n}$ is the largest zero of $x^{m+n}-x^{m}-x^{n}-1$.

ProOF. By Lemma 1.3 and 1.9(2), it suffices to prove $h(g) \geq \log \mu_{m, n^{\prime}}$ for $g$ satisfying (1), (2) and (3) of Lemma 1.3. Let $G$ be the $A$-graph of $g$ with respect to the two orbits of period $m$ and $n^{\prime}$. Then, by Lemmas 1.4 and $1.5, G$ has a subgraph $G^{\prime}$ which has $s$ vertices and just three simple loops $L_{1}, L_{2}$ and $L_{3}$ all through $J$ of length $l_{1}, l_{2}$ and $l_{3}$ respectively such that $l_{1} \leq l_{2} \leq l_{3}, l_{1} \leq \min \left\{m, n^{\prime}\right\}, l_{2} \leq$ $\max \left\{m, n^{\prime}\right\}$ and $l_{3} \leq m+n^{\prime}$. By Lemma 1.8, $h\left(G^{\prime}\right)=\log \nu$ where $\nu$ is the largest zero of $x^{s}-x^{s-l_{1}}-x^{s-l_{2}}-x^{s-l_{3}}$, i.e. that of $x^{l_{3}}-x^{l_{3}-l_{1}}-x^{l_{3}-l_{2}}-1$. By Lemma 1.9(1), we have $\mu_{m, n^{\prime}} \leq \nu$ and by Lemmas 1.6 and 1.7,

$$
h(g) \geq h(G) \geq h\left(G^{\prime}\right) .
$$

Therefore $h(g) \geq \log \mu_{m, n^{\prime}}$. 
2. In this section we shall prove Theorem B stated in the introduction. First we need several lemmas from [3].

LEMMA 2.1 [3, THEOREM 3.2]. If $f$ is an endomorphism of the circle of $|\operatorname{deg} f| \leq$ $1, F$ is a lifting of $f$ and $F$ has a periodic point of period $m>1$, then $h(f) \geq$ $\left(\log \lambda_{p}\right) / 2^{k}$ if $m=2^{k} \cdot p, p$ is odd and $p>1$, where $\lambda_{p}$ is the largest zero of $x^{p}-$ $2 x^{p-2}-1$.

LEMMA 2.2 [3, Proposition 3.4]. Let $\operatorname{deg} f=-1$ and let $x \in R$ be a point such that $\pi(x)$ is a periodic point of $f$ of period $m, m$ odd. Then there exists a lifting $F$ of $f$ such that $x$ is a periodic point of $F$ of period $m$.

The next lemma is trivial.

LEMMA 2.3. The largest zero $\lambda_{m}$ of $x^{m}-2 x^{m-2}-1$ is larger than the largest zero $\mu_{m, n}$ of $x^{m+n}-x^{m}-x^{n}-1$ where $m \geq 2$ and $n \geq 2$.

The following is one of the main theorems of [3].

LEMMA 2.4 [3, THEOREM 3.9]. Let $f$ be an endomorphism of $S$ of degree 1. Let $f$ have a fixed point $x$ and a periodic point $y$ of period $n>1$ such that $\rho(F, x) \neq$ $\rho(F, y)$ where $F$ is a lifting of $f$. Then $h(f) \geq \log \mu_{n}$ where $\mu_{n}$ is the largest zero of the polynomial $x^{n+1}-x^{n}-x-1$.

Proof of THEOREM B. (1) $\operatorname{deg} f=1$. If $f$ has no fixed point, then this theorem is a special case of Theorem $A$ which we have proved in $\S 1$. If $f$ has a fixed point $x$, then we may choose a lifting $F$ of $f$ such that $\rho(F, x)=0$. Let $a$ and $b$ be periodic points of $f$ of period $m$ and $n$ respectively such that $m \geq 2, n \geq 2$ and $(m, n)=1$. If $\rho(F, a)=\rho(F, b)=0$, then $a$ and $b$ are also periodic points of $F$ of period $m$ and $n$. As $(m, n)=1$, we may assume $m$ is odd and $m \geq 3$. Then, by Lemmas 2.1 and 2.3 we have $h(f) \geq \log \lambda_{m}>\log \mu_{m, n}$. If either $\rho(F, a) \neq 0$ or $\rho(F, b) \neq 0$, then by Lemma $2.4, h(f) \geq \log \mu_{m}$ or $h(f) \geq \log \mu_{n}$. On the other hand, by Lemma 1.9(2), $\min \left\{\mu_{m}, \mu_{n}\right\} \geq \mu_{m, n}$. Therefore $h(f) \geq \log \mu_{m, n}$.

(2) $\operatorname{deg} f=-1$. We may assume $m$ is odd and $m \geq 3$. By Lemmas 2.2, 2.1 and 2.3, $h(f) \geq \log \lambda_{m}>\log \mu_{m, n}$.

(3) $\operatorname{deg} f=0$. We may assume $m$ is odd and $m \geq 3$. In this case, given any periodic point of $f, F$ has a periodic point of the same period (see [3, Proposition 3.3]). therefore, by Lemmas 2.1 and 2.3, we have $h(f) \geq \log \lambda_{m}>\log \mu_{m, n}$.

(4) $|\operatorname{deg} f| \geq 2$. $h(f) \geq \log |\operatorname{deg} f| \geq \log 2>\log \mu_{m, n}$.

Finally we give an example to show our estimate of the smallest entropy in Theorem $B$ is the best possible.

EXAMPLE. Let $m$ and $n$ be positive integers such that $1<m<n$ and $(m, n)=$

1. Then there exist $p$ and $q \in N$ such that $p<m, q<n$ and $n p-m q=1$.

Let us define $F: R \rightarrow R$ as the following.

$$
F(x)= \begin{cases}x+\frac{q}{n}, & 0 \leq x \leq 1-\frac{1}{n} \\ \frac{2 m-1}{m-1}\left(x-\frac{n-1}{n}\right)+\frac{q-1}{n}+1, & 1-\frac{1}{n} \leq x \leq 1-\frac{1}{m n} \\ (1-m)(x-1)+\frac{q}{n}+1, & 1-\frac{1}{m n} \leq x \leq 1\end{cases}
$$

and $F(x+k)=F(x)+k$ for $k \in Z$. 
This is a piecewise linear function obtained by modifying $x+q / n$ slightly to assume a maximum $1+(q+1) / n-1 / m n$ at $x=1-1 / m n$. Let $f(x)=\pi F(x) \mid[0,1]$, then it is an endomorphism of $S$ of degree 1 and has periodic points $\pi(j q / n), j=$ $0, \ldots, n-1$, of period $n$ and $\pi((i q+1) / n-1 / m n), i=1, \ldots, m$, of period $m$. Let $G$ be the $A$-graph of $f$ with respect to these periodic points of period $m$ and $n$ shown above. Then it is not difficult to see $h(f)=h(G)=\log \mu_{m, n}$.

\section{REFERENCES}

1. C. Bernhardt, Rotation intervals of endomorphisms of the circle, $\mathrm{Ph}$. D. Thesis, University of Warwick, 1980.

2. L. Block, E. M. Coven and Z. Nitecki, Minimizing topological entropy for maps of the circle, preprint.

3. L. Block, J. Guckenheimer, M. Misiurewicz and L. S. Young, Periodic points and topological entropy of one dimensional maps, Global Theory of Dynamical Systems, Proceedings, Northwestern Univ., 1979, Lecture Notes in Math., vol. 819, Springer-Verlag, Berlin and New York, 1980, pp. 18-34.

4. R. Ito, Rotation sets are closed, Math. Proc. Cambridge Philos. Soc. 89 (1981), 107-111.

5. M. Misiurewicz and W. Szlenk, Entropy of piecewise monotone mappings, Studia Math. 67 (1980), 45-63.

6. S. Newhouse, J. Palis and F. Takens, Stable families of dynamical systems. I: Diffeomorphisms, preprint, I.M.P.A., Rio, Brazil.

DEPARTMENT OF MATHEMATICS, SCHOOL OF EDUCATION, WASEDA UNIVERSITY, SHINJUKU, TOKYO, JAPAN 